\title{
Protective Effect of Natural Products against Chemotherapy- Induced Cardiotoxicity: A Review
}

\author{
Siti Nurul Najiha Othman' ${ }^{1}$ Pei Teng Lum ${ }^{1}$, Siew Hua Gan², Shankar Mani ${ }^{3}$, Mahendran Sekar ${ }^{1, *}$
}

\section{Siti Nurul Najiha Othman ${ }^{1}$, Pei Teng Lum', Siew Hua Gan², Shankar Mani ${ }^{3}$, Mahendran Sekar ${ }^{1, *}$}

'Department of Pharmaceutical Chemistry, Faculty of Pharmacy and Health Sciences, Universiti Kuala Lumpur Royal College of Medicine Perak, Ipoh - 30450, Perak, MALAYSIA.

${ }^{2} S$ chool of Pharmacy, Monash University Malaysia, Bandar Sunway 47500, Selangor Darul Ehsan, MALAYSIA.

${ }^{3}$ Department of Pharmaceutical Chemistry, Sri Adichunchanagiri College of Pharmacy, Adichunchanagiri University, BG Nagara, Nagamangala, Mandya-571418, Karnataka, INDIA.

\section{Correspondence}

\section{Assoc. Prof. Dr. Mahendran Sekar}

Department of Pharmaceutical Chemistry, Faculty of Pharmacy and Health Sciences, Universiti Kuala Lumpur Royal College of Medicine Perak, Ipoh - 30450, Perak MALAYSIA.

Phone no: (6016) - 3346653

Fax: (605) - 2536634

E-mail: mahendransekar@unikl.edu.my History

- Submission Date: 30-04-2020;

- Review completed: 20-05-2020;

- Accepted Date: 03-06-2020

DOI : 10.5530/pj.2020.12.166

Article Available online

http://www.phcogj.com/v12/i5

\section{Copyright}

(C) 2020 Phcogj.Com. This is an openaccess article distributed under the term of the Creative Commons Attribution 4.0 International license.

\section{ABSTRACT}

Background: Cancer is one of the diseases with high mortality rate recorded each year across the world. Its mainstay treatment is chemotherapy although they are largely toxic, causing severe adverse reactions including cardiotoxicity, nephrotoxicity and genotoxicity. Cardiotoxicity is unique to certain chemotherapeutic agents and occur via several mechanisms. It has been hypothesized that co-administration of natural products which may be cardioprotectant, together with chemotherapy can alleviate cardiotoxicity-induced by chemotherapy. Objectives: This review aimed to provide a brief information about the protective effect of natural products against chemotherapy-induced cardiotoxicity Methods: To complete this review, relevant literatures were searched from several scientific databases including Google, Google Scholar, Scopus, Web of Science and Pubmed. Results: In this paper, we have reviewed ten natural products (curcumin, mangiferin, naringenin, quercetin, 6-gingerol, lycopene, resveratrol, apigenin, proanthocyanidins and indole-3-carbinol), which have major influences in attenuating chemotherapy-drug induced cardiotoxicity. Apart from the cardioprotective effects, they tend to confer some synergistic effects with chemotherapeutic agents and therefore have the potential to be used as an adjunct. Conclusion: Though a panel of natural products demonstrate protective effects against cardiotoxicity in cells and animal models, their therapeutic potentials for clinical needs further investigation.

Key words: Chemotherapy, Anticancer drugs, Cardiotoxicity, Natural products, Cardioprotective.

\section{INTRODUCTION}

Chemotherapy is the main treatment strategies for cancer patients which may be used alone or in combination with surgery and/or radiotherapy. Chemotherapy is the delivery of cytotoxic agents that have cell-killing activities in order to reduce tumor size or any residuals which creates extensive side effects. ${ }^{1}$ These side effects include general cell-damaging effects including decreased blood cells, myelosuppression and immunosuppression. More specific side effects are nephrotoxicity, neurotoxicity, testicular dysfunction, cardiotoxicity and hepatotoxicity. ${ }^{2-4}$ Nevertheless, problems such as myocardial toxicity, ischemia and arrhythmia may arise with the usage of certain anticancer treatment such as anthracyclines and 5-fluorouracil. ${ }^{5}$ Among all the cardiotoxicity events, cardiac dysfunction and heart failure are reported to be among the most serious cardiovascular disease due to the administration of systemic cancer drugs like anthracyclines and 5-fluorouracil which can be acute or delayed response. ${ }^{5,6}$

In the past, cardiotoxicity was less common. However, its incidence is increasing, contributed by the use of combination therapy and adjuvant treatment. ${ }^{7}$ Common anticancer drugs that cause cardiovascular disease are doxorubicin and epirubicin from the anthracycline family, paclitaxel and docetaxel (plant alkaloids), cyclophosphamide (alkylating agent) and other drugs from other classes including 5 -fluorouracil. ${ }^{5}$
To date, there is a great interest in natural products due to their purported reduced side effects. There is also a trend of combining various anticancer drugs with natural products, aiming at maximizing their efficacies while minimizing cardiotoxicity through the delivery of lower doses of chemotherapies. These main source of such natural products are mainly obtained from commonly available fruits and vegetables (Figure 1). Hence, in this review, we discuss on ten natural products includes curcumin, mangiferin, naringenin, quercetin, 6-gingerol, lycopene, resveratrol, apigenin, proanthocyanidins and indole-3-carbinol (Figure 2), reported to play major roles in attenuating chemotherapy-induced cardiotoxicity.

\section{METHODS}

To complete this review, relevant literatures were searched from several scientific databases including Google, Google Scholar, Scopus, Web of Science and Pubmed. The categories of keywords used for searching are "Natural Products" or "Phytoconstituents" or "Isolated Compounds" or "Natural Compounds" or "Curcumin" or "Mangiferin" or "Naringenin" or "Quercetin" or "6-Gingerol" or "Lycopene" or "Resveratrol" or "Apigenin" or "Proanthocyanidins" or "Indole-3carbinol" and "Cardioprotection" or "Anticancer" or "Chemotherapy" or "Cardiotoxicity" or "Cardiotonic". After screening of literatures from 2005 to 2019, a total of ten natural products investigated the protective effect against chemotherapy-induced cardiotoxicity and that were included in the present review.
Cite this article: Othman SNN, Lum PT, Gan SH, Mani S, Sekar M. Protective Effect of Natural Products against Chemotherapy-Induced Cardiotoxicity: A Review. Pharmacogn J. 2020;12(5):1180-9. 


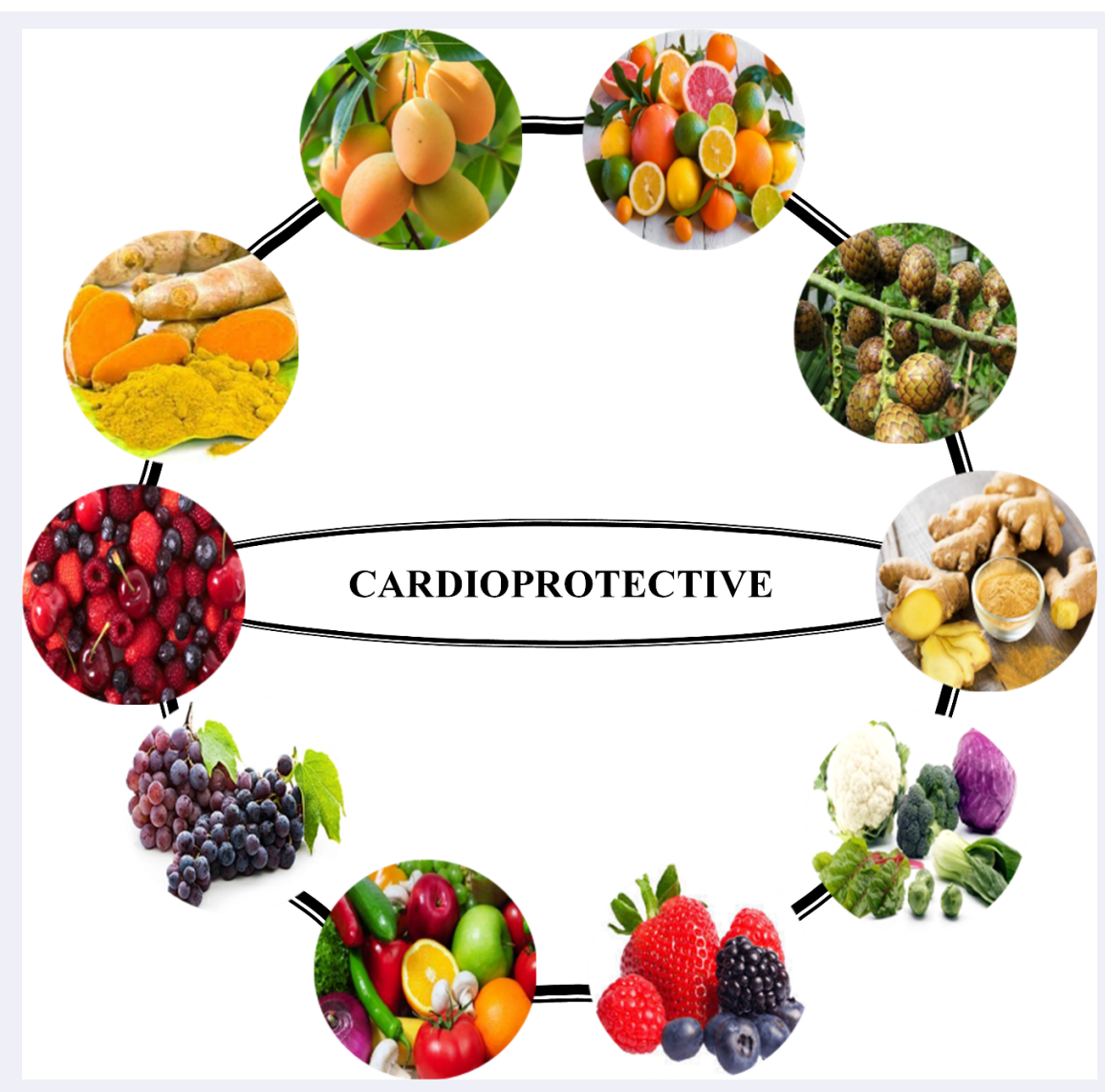

Figure 1: Source of natural products protective against chemotherapy-induced cardiotoxicity.<smiles>COC1CC(CCC(O)CC(O)CCC2CCC(O)C(O)C2)CCC1O</smiles>

Curcumin<smiles>OC[C@H]1OC(C2C(O)CC3OC4CC(O)C(O)CC4C(O)C3C2O)[C@H](O)[C@@H](O)[C@@H]1O</smiles>

Mangiferin<smiles>OC1CCC(C2CC(O)C3C(O)CC(O)CC3O2)CC1</smiles>

Naringenin<smiles>CCCCCC(O)CC(O)CCC1CCC(O)C(OC)C1</smiles><smiles>OC1CCC(CCC2CCC(O)C(C3CC(O)CC(O)C3C3CC(O)CC(O)C3)C2)CC1</smiles><smiles>CC(C)CCCC(C)CCCC(C)CCCC(C)CCCCC(C)CCCC(C)CCCC(C)CCCC(C)C</smiles><smiles>OCC1CNC2CCCCC12</smiles>

Indole-3-Carbinol

Figure 2: Natural products protective against chemotherapy-induced cardiotoxicity. 


\section{PROTECTIVE EFFECT OF NATURAL PRODUCTS AGAINST CHEMOTHERAPY-INDUCED CARDIO- TOXICITY}

\section{Curcumin}

Curcumin is a naturally occurring chemical found in turmeric (curcuma longa), natively found in the South and Southeast Tropical Asia. When the tuber is grinded, the orange-yellow crystalline powder insoluble in water with a chemical structure of $\mathrm{C}_{21} \mathrm{H}_{20} \mathrm{O}_{6}$ is formed. ${ }^{8}$ Since the Ayurvedic period (1900 b.c.), turmeric has been widely applied in the treatment of various ailments including skin, pulmonary, gastric systems, pains, wounds and liver problems. ${ }^{9}$ In various in-vitro and in-vivo studies, the compound have been confirmed to have good anti-inflammatory, antioxidant, wound healing and anticancer properties. ${ }^{9}$

Curcumin can reduce atrophy of the myocardium as well as fibrosis of the cardiac cells. ${ }^{10}$ It also decreases the apoptotic myocytes occurring in mice treated with doxorubicin. Interestingly, curcumin is said to exhibit cardioprotective effect when co-administered with doxorubicin which may be contributed by autophagy. ${ }^{10}$ In fact, it has been hypothesized that repeated administration of doxorubicin reduce antioxidant level which increase the levels of cardiac biomarkers at the same time, giving the overall effect of inducing cardiomyopathy. Overall, the mechanism of cardiotoxicity as induced by doxorubicin are several including oxidative damage level increase, increased in inflammation and apoptosis. However, administration of curcumin ( $1 \mathrm{mg} / \mathrm{kg}$, i.p., 5 days $)$ prior to doxorubicin $(20 \mathrm{mg} / \mathrm{kg}$, i.p.) may help protect the myocardium where significant reduction in biomarkers including lactate dehydrogenase $(\mathrm{LDH})$, creatine phosphokinase $(\mathrm{CPK})$, aspartate aminotransferase (AST), alanine transaminase (ALT) and alkaline phosphate (ALP) were seen. At the same time, glutathione, superoxide dismutase and catalase levels were increased while elevated levels of malondialdehyde tend to be ameliorated. ${ }^{11}$

When administered orally for 14 days, both curcumin aqueous and ethanolic extracts ( $200 \mathrm{mg} / \mathrm{kg}$, p.o.) have the ability to reduce mortality rate of rats which received doxorubicin $(15 \mathrm{mg} / \mathrm{kg}$, i.p.). Besides lowering $\mathrm{LDH}$ and MDA levels, it also ameliorates creatine-kinase MB (CK-MB), serum nitric oxide and calcium levels in the heart. Together, the levels of ascorbic acid and glutathione in the heart was found to increase significantly. ${ }^{12}$ Sadzuka et al. ${ }^{13}$ further confirmed that curcumin (10 and $100 \mathrm{mg} / \mathrm{kg}$, i.p.) can reduce the adverse effects related to doxorubicin ( $2 \mathrm{mg} / \mathrm{kg}$, i.p.) since both combination can help overcome glutathione peroxidase activity reduction and improve lipid peroxidation in the heart muscles indicating that it has some cardioprotective effect.

Cyclophosphamide is also an antineoplastic agent that may cause cardiac problem such as interstitial edema and hemorrhage, mainly attributed to the cause of oxidative stress. Cyclophosphamide induced oxidative stress results in abnormal cell death and these toxic metabolites lead to a direct damage of endothelial capillary blood vessels. ${ }^{14}$ In a report, a group of rats treated with curcumin $(100 \mathrm{mg} /$ $\mathrm{kg}$, p.o.) together with cyclophosphamide $(30 \mathrm{mg} / \mathrm{kg}$, i.p.) had low levels of damage in the myocardium based on histopathological and immunohistochemical properties as compared to rats administered with cyclophosphamide alone. ${ }^{14}$ Based on the study by Chakraborty et al. ${ }^{15}$, the combination of oral curcumin $(50 \mathrm{mg} / \mathrm{kg}$, p.o.) and piperine (20 mg/kg, p.o.) confer better protection against cardiac toxicity that is induced by cyclophosphamide $(200 \mathrm{mg} / \mathrm{kg}$, i.p.) as compared to treatment with curcumin $(200 \mathrm{mg} / \mathrm{kg}$, p.o. $)$ alone. ${ }^{15}$ In another study, pretreatment of curcumin $(200 \mathrm{mg} / \mathrm{kg}$, p.o.) for 7 days before cisplatin ( $5 \mathrm{mg} / \mathrm{kg}$, i.p.) exhibit cardioprotective effect based on histopathology, biochemical and cytokines changes when compared with cisplatinalone treated group. ${ }^{16}$

\section{Mangiferin}

Mangiferin, chemically known as 2-C- $\beta$-D-glucotranosyl-1,3,6,7tetrahydroxyxanthen-9-one with molecular formula of $\mathrm{C}_{19} \mathrm{H}_{18} \mathrm{O}_{11}$ is a phytochemical, abundant in the fruits and leaves of the mango plant. Mangiferin, which appears as a yellow crystalline powder isolated from Mangifera indica have been widely used as a traditional medicine. It has various pharmacological activities including as antioxidant, antibacterial, antidiabetic, tumor suppression ability and anticancer effects. ${ }^{17,18}$ Interestingly, the phytochemical compound has the ability to protect against various types of cancers in human including lung, colon, breast and neuronal cancers. ${ }^{19}$

Mangiferin can prevent elevation of the biomarkers such as $\mathrm{LDH}$, AST, ALT and ALP due to doxorubicin-induced cardiotoxicity in rats. Apart from that, the imbalance of glutathione reductase, glutathione peroxides, superoxide dismutase, glutathione and lipid profiles as a result of doxorubicin administration can be improved. ${ }^{20}$ Furthermore, mangiferin has the ability to normalize serum calcium homeostasis, as shown on the rats' SERCA2a mRNA expression level via activation of intrinsic apoptotic pathways where intracellular calcium regulation is part of the cardioprotective mechanism shown. ${ }^{21}$

Bhatt et al. ${ }^{22}$ confirmed the cardioprotective ability and inhibition of cardiac stress conferred by doxorubicin by the ethanolic extract of Mangifera indica. Rats which received the extract alone resulted in decrease in serum cardiac biomarker and improvement in antioxidant levels in cardiac tissues. Rats that received doxorubicin $(15 \mathrm{mg} / \mathrm{kg}$, i.p.) and mangiferin leaf alcoholic extract $(100 \mathrm{mg} / \mathrm{kg}$, p.o.) for 21 days showed improved lipid profiles, electrocardiographic parameters, histologic score and have lower mortality rates. ${ }^{23}$ Additionally, there was a decrease in the biomarker enzyme levels and an increase in the antioxidant level in tissues of animals which received mangiferin. Rats which received cyclophosphamide and mangiferin combination also showed better lipid profile, electrocardiogram parameters, histology scores and had longer survival rates. ${ }^{22}$ Apart from alleviating the cardiac toxicity caused by anticancer drugs, mangiferin is also said to protect against isoproterenol-induced cardiotoxicity due to its high antioxidant activity. $^{24}$

\section{Naringenin}

Naringenin is mainly found in citrus fruits and vegetables from the flavanones class of flavonoids such as in lemon, orange, tangerine and grapefruit. ${ }^{25,26}$ It is reported to be effective in treating inflammation, fibrosis, sepsis and cancer. To date, its pharmacological activity has been confirmed in various animal models where it has shown antioxidant, antitumor, antiviral, antibacterial and cardioprotective abilities. ${ }^{27,28}$

Doxorubicin can enhance the production of inflammatory biomarkers such as transforming growth factor $\beta 1$ (TGF- $\beta 1$ ), tumor necrosis factor alfa (TNF- $\alpha$ ), interleukin-6 (IL-6) and interleukin-10 (IL-10). Coadministration of doxorubicin $(3 \mathrm{mg} / \mathrm{kg}$, i.p.) and naringenin $(50 \mathrm{mg} /$ $\mathrm{kg}$, p.o.) however, confers less damaging effects on the cardiac muscles as confirmed histologically while returning the mRNA expression of the biomarkers back to normalcy. ${ }^{29}$ It has been reported that pretreatment of naringenin (25 mg/kg, p.o.) to male Swiss albino rats decreases creatinine phosphokinase by $57 \%$ as compared to doxorubicin-only group. Additionally, glutathione S-transferase, catalase and sodium dismutase levels also increase by more than $90 \%$ as compared to doxorubicin-only group indicating that naringenin may be a useful adjunct to be administered with doxorubicin. ${ }^{30}$

Xiuzhen et al. ${ }^{31}$ reported that naringenin-7-O-glucoside isolated from Dracocephalum rupestre which is a rhizotomos herb can inhibit cardiomyocyte apoptosis and has some cardioprotective activity. Another important constituent in the herb is naringenin which can 
improve the mRNA expression of glutamate-cysteine ligase modifier as well as glutamate-cysteine ligase subunit. The researchers also concluded that doxorubicin-induced cardiomyocytes can be inhibited by naringenin administration $(10,20$, and $40 \mu \mathrm{M})$ since the constituent enhances antioxidant enzymes by phosphorylation of extracellular signal-regulated kinases $1 / 2($ ERK1/2) and also nuclear translocation of nuclear factor erythroid 2-related factor 2 (Nrf2). ${ }^{31}$ Additionally, the restoration of antioxidant content and mitochondrial activities are also important contributing factors for the protective effect conferred by naringenin. Histopathological findings confirmed that naringenin prevents myofibrillar loss, inflammation and interstitial edema in the heart. When administered at two different doses (50 and $100 \mathrm{mg} / \mathrm{kg}$ ), naringenin confers protection against mitochondrial dysfunction while preserving myocytes function at the same time. ${ }^{32}$

It has been reported that naringenin protects against doxorubicininduced cardiotoxicity without affecting cancer cells. It reduced the levels of glutamic pyruvic transaminase (GPT), glutamic oxaloacetic transaminase (GOT), CK-MB and $\mathrm{LDH}$ when naringenin was administered (50 and $100 \mathrm{mg} / \mathrm{kg}$, i.p., 14 days) prior to doxorubicin treatment. Additionally, there was significant reduction in DOXinduced 8-OHdG DNA adducts and Poly (ADP-ribose) polymerase PARP activities with enhancements in blood antioxidant levels seen. ${ }^{33}$

\section{Quercetin}

Quercetin is a flavonoid found in various plants including Camellia sinensis, Calamus scipionum and Moringa oleifera. ${ }^{34}$ Its IUPAC nomenclature is $3,3^{\prime}, 4^{\prime}, 5,7$-pentahydroxyflvanone or 3,3', $4^{\prime}, 5,7$-pentahydroxy-2-phenylchromen-4-one. ${ }^{35} \quad$ Quercetin contains five phenol functional groups which has amphoteric characteristics and is found not only in tea but in fruits, vegetables and wine. ${ }^{35-36}$ However, the compound has low solubility, making its bioavailability a challenge although the bioflavonoid is useful in alleviating many human ailments. ${ }^{37}$ Additionally, quercetin is also said to be useful in combating inflammation and improving the immune system. Another group of researchers stated that quercetin can help reduce the risk of cardiovascular diseases, metabolic problems as well as cancer due to its high antioxidant nature. ${ }^{34}$ Apart from that, it also exhibits antiviral activities, help in reducing lipid peroxidation, aggregation of platelet and permeability of the blood capillary. ${ }^{36}$

In an experiment that utilised quercetin to be administered $(50 \mathrm{mg} /$ $\mathrm{kg}$, p.o., 15 days) prior to and during doxorubicin treatment, there was significant cardioprotective activity where there were reduction in troponin, CK-MB count, creatine phosphokinase. ${ }^{38}$ Quercetin may also help by stabilizing enzyme levels including AST, ALT, ALP, LDH and creatine to normalcy. It was stated that its high antioxidant content helped in retaining serum enzyme levels as well as reducing cell damage and protect the heart. ${ }^{39}$

Quercetin is also said to confer some cardioprotective effect by strengthening the mitochondrial function in cardiomyocytes. Doxorubicin harms cardiac cells by interrupting with the 14-3$3 \gamma$ expression regulation. When quercetin is administered prior to doxorubicin treatment, there was better cell viability, improved catalase, SOD, GSH levels and at the same time decreases caspase-3-activities as well as reactive oxygen species (ROS). Mechanistically, it was said to knock down the 14-3-3 $\gamma$ expression via pAD/14-3-3 $\gamma$-shRNA. Thus, suppression of oxidative stress and enhanced mitochondrial function is believed to be another mechanism of its cardioprotective effect. ${ }^{40}$

The creation of combined hydrophobic quercetin with hydrophilic doxorubicin by using biocompatible nanocarrier via amphiphilic polymer has so far been successful since nanoparticles can protect vascular endothelial cells from cardiac cytotoxicity conferred by doxorubicin. The compound is said to kill cancer cells while maintaining serum cardiac levels and cardiac function as well as repairing the damages in cardiac tissues. ${ }^{41}$ Better ventricular ejection fraction and normal creatine kinase was exhibited among mice treated with a combination of quercetin and resveratrol micelles. Additionally, doxorubicin dosing can be reduced through chemosensitization as it improved the effectiveness of treatment while protecting the damaging effect on the cardiovascular system. ${ }^{42}$ Formulation of quercetin nanoparticles by using various methods and solvents is said to confer better cardioprotection due to better release of quercetin particles to the targets. Nevertheless, nanoparticles formulation of quercetin shows better therapeutic response without interrupting the activity of the

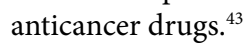

\section{6-Gingerol}

6-Gingerol is a major biochemical compound isolated from ginger, Zingiber officinale which is from the Zingiberaceae family. It is one of the perennial herbs that can easily be found in Malaysia and throughout Asia. ${ }^{44}$ The rhizome have been widely used for spice condiments as a flavour enhancer in dishes and also for medicinal purposes for more than 20 years. The compound which emits a pungent smell has multiple health benefits. ${ }^{45}$ For example, 6-gingerol is a ketone type chemical compound has limitations to be used as medicine due to its insolubility in water although the compound is very useful in combating various human ailments such as tumor, hypertension and inflammation. ${ }^{46}$ It is also biologically active as anticoagulant, antioxidant and antitumor. ${ }^{46}$ The compound is generally used in the treatment and prevention of various chronic diseases. ${ }^{45}$

Aqueous and ethanolic extract of ginger have been reported to ameliorate doxorubicin-induced cardiotoxicity in rats, due to its positive effect in ameliorating oxidative stress. ${ }^{47}$ The combination of oral ginger and cisplatin showed 1) improved cardiac histological studies and structures 2) reduction in the activity of P53 and TNF- $\alpha$ immuneexpressions and 3) reduced blood CK and LDH levels. The cisplatin alone-treated group showed disorganized cardiac muscle fibers appear, interrupted myofibrils, inflamed mitochondria, widen intercalated discs and elevated serum CK and LDH levels shown. ${ }^{48}$

Additionally, cyclophosphamide treatment caused increased troponin I levels and increased levels of oxidative markers. However, no abnormality in troponin I and oxidative markers levels were seen in adult male albino rats that were treated with ginger and cyclophosphamide combination. Histological findings confirmed that the disruption in cardiomyocytes cytoplasm seen in cyclophosphamide alone-treated rats did not happen with ginger and cyclophosphamide combination. ${ }^{49}$ In another study, 6-gingerol and doxorubicin combination were found to have vascular protection which negates doxorubicin toxicity effect on the heart. Besides giving protection on the blood vessels, 6-gingerol acts synergistically with chemotherapy against cancer cells. ${ }^{50}$

In another report, chronic cardiomyopathy induced by doxorubicin was ameliorated by 6-gingerol occuring via 1) inhibition of myocardial ROS production 2) reduction of inflammation by attenuation of NF- $\mathrm{kB}$ activity 3 ) down-regulation of soluble receptor for advance glycation end product (sRAGE) and 4) interruption of myocardial apoptosis as a result of increased caspase-3 activities. ${ }^{51}$ Combination of 6-gingerol with higenamine which is the main active ingredients of Sini decoction, a traditional and complementary medicine is said to be cardioprotective, due to reactivation of phosphatidylinositol-3-kinase ( $\left.\mathrm{PI}_{3} \mathrm{~K} / \mathrm{Akt}\right)$ signaling pathways. These two active ingredients itself confers some protection against cardiomyocytes which was related with mitochondria-dependent apoptosis process. ${ }^{52}$

\section{Lycopene}

Lycopene is categorized as unsaturated carotenoid which can easily be found in red fruits and vegetables such as tomato, watermelon, 
grapefruits and guava, giving their red color. The hydrocarbon carotenoids has the molecular formula of $\mathrm{C}_{40} \mathrm{H}_{56}$ with an acyclic open chain structure containing 13 double bonds. It has lipophilic characteristic which contribute to its ability to soluble in ethanol, methanol and water. Lycopene levels follow the stages of fruit ripening. ${ }^{53}$ In addition, the levels are also affected by climatic changes, geographical location, species and environmental conditions. ${ }^{54}$

Lycopene is believed to be the most potent carotenoid antioxidant when investigated in-vitro where it has shown good anti-inflammatory activity and protective effects on the cardiovascular system, ameliorating hypertension and metabolic syndrome. ${ }^{55}$ It has also been hypothesized to have antiplatelet, anti-apoptotic and anti-atherosclerotic effects. ${ }^{53}$

Mozos et al..$^{53}$ stated that lycopene can enhance vascular function and is therefore beneficial in preventing cardiovascular disease. Apart from its natural ability to regulate blood pressure to normalcy level, lycopene also has cardioprotective effect against doxorubicin toxicity which is attributed to 1) its antioxidant content 2) the ability to form foam cells and 3) protect against cell damage of endothelial cells. ${ }^{53}$ Low density lipoproteins (LDL) undergoes oxidation to initiate atherosclerosis which can promote cardiovascular disease progression. Therefore, lycopene is useful for the elderly, smokers, diabetics, hemodialysis and patients with acute myocardial infarction. Additionally, combination of Lycopene with other active compounds is beneficial in protecting cardiovascular health..$^{55}$

Lycopene-loaded liposomes which have smaller and uniform particle sizes have been designed to improve the effectiveness of its delivery in order to enhance doxorubicin effects while decreasing its cardiotoxicity, thus leading to increased cytotoxic effect and reduction in tumor. ${ }^{56}$ Alleviation of cardiotoxicity as induced by doxorubicin may be due to its mechanism of 1) quenching singlet oxygen, peroxy radicals, free radicals reaction 2) restoration of vitamins $C$ and $E$ levels 3 ) reduction in DNA damage 4) restoration of cellular antioxidant and 5) avoidance of glutathione depletion. Ojha et al..$^{51}$ further confirms that lycopene can protect the heart against cardiac oxidative DNA damage caused by doxorubicin single treatment due to its antioxidant action.

Another study investigated the effects of lycopene and saffron on doxorubicin-induced cardiac via histological and biochemical assessments on adult male albino rats. The researchers concluded that co-administration of doxorubicin and lycopene improved all investigated parameters including cardiac enzymes and troponin levels. However, no study have been confirmed in indicating that more clinical studies are required. ${ }^{57}$

\section{Resveratrol}

Resveratrol is a polyphenol that are naturally produced by some plants as part of its self-defence mechanism. Grapes, wine and peanuts are the plants that have high amounts of the said polyphenol. Chemically named as 3,5,4'-trihydroxystilbene, it has a stilbene group which are two aromatic rings joined together by a methylene bridge. Resveratol appeared in two different isomers which are trans-resveratrol and cis-resveratrol; the former has a higher biological activity which is contributed by the 4 '-hydroxystyryl group. ${ }^{58}$

Resveratrol provides a wide range of medical benefits including cardioprotection, anti-inflammatory activity and antiplatelet properties. ${ }^{58}$ Clinical trials have focused on using resveratrol to treat cancer, brain and neuron problems, cardiac problems, diabetes and obesity with large improvement seen in diabetes, neurological and cardiovascular problems. However, its poor bioavailability limits its clinical benefits. ${ }^{59}$

Doxorubicin affects H9C2 cells apoptosis due to inhibition of AMPactivated protein kinase (AMPK) activation and enhancement in pro-apoptotic protein expression. Nevertheless, co-administration of resveratrol and doxorubicin caused a reduction in apoptotic cells ratio and improvement in autophagy ratio due to improved autophagy via AMPK/ mammalian target of rapamycin (mTOR)/ serine/threonine protein kinase pathway (ULk1) or in short, AMPK/mTOR/ULk1 pathway. Gu et al. ${ }^{60}$ stated that resveratrol $(10 \mathrm{mg} / \mathrm{kg}$, i.p., 7 days $)$ and doxorubicin (15 mg/kg, i.p., 7 days) combination is more effective as compared to doxorubicin alone since resveratrol (10 mg/kg, i.p., 7 days) exhibits its antioxidant effect by ameliorating doxorubicin-induced oxidative stress via enhancement of antioxidant enzyme activities including superoxide dismutase and catalase.

Apart from enhanced cardiac protection activity by mitigating cancer cell apoptosis as induced by doxorubicin, administration of resveratrol (10 mg/kg, i.p., 8 days) can also inhibit oxidative stress caused by cyclophosphamide. ${ }^{61}$ Moreover, resveratrol not only enhances cytotoxic activity of anticancer drugs, but can help protect cardiac cells by defending the cardiomyocytes against apoptosis. AMPK/ P53 pathway is an important mechanism in influencing doxorubicininduced cardiac cells death. Nevertheless, resveratrol can enhance AMPK phosphorylation and inhibit P53 expression, at the same time downregulating Bax expression, all of which are positive effects on the heart. $^{62}$

In another study by Alanazi et al. ${ }^{63}$, treatment with resveratrol liposome, ameliorates all the biochemical and histological changes inflicted by anticancer drugs. Serum CK-MB, troponin-I and LDH returned to normalcy as compared to the group of animals which received doxorubicin treatment only. Additionally, there were improvement in cardiac antioxidant levels as well as in the expression of S100 calcium binding protein A1 (S100A1) and sarcoplasmic/endoplasmic reticulum calcium ions ATPase 2a (SERCA2a). ${ }^{63}$ Experimental design using solid nanoparticles loaded with resveratrol which is poorly soluble in water (thus limiting its efficacy) shows better heart rate, ejection fractions, fractions shortening and degree of myocardial lesions on mice. Thus, resveratrol is believed to be a promising phytochemical compound to be co-utilised with anticancer treatment to help alleviate adverse reactions of cytotoxic drugs. ${ }^{64}$

In another experimental design using juvenile mouse model of doxorubicin-induced cardiotoxicity, there were confirmed impediment in the ability of the heart to adapt to hypertension in the presence of doxorubicin. Co-administration of resveratrol $(0.4 \%, \sim 320 \mathrm{mg} / \mathrm{kg} / \mathrm{day}$, 7 days) with doxorubicin $(4 \mathrm{mg} / \mathrm{kg}$, i.p. $)$ treatment however, showed normalized molecular biomarkers of cardiotoxicity. It also repairs and restores the heart to allow adaptation and better response to hypertension. ${ }^{65}$

\section{Apigenin}

Apigenin is an active plant-derived flavone found in a wide range of common fruits, beverages and vegetables. The compound is present in foods normally as glucoside conjugates and other acylated derivatives that are water-soluble when compared to its parent compound. ${ }^{66}$ Apigenin is abundant in citrus fruits such as oranges, onions, maize, rice, tea and parsley. It is chemically known as 4',5,7-trihydroxyflavone with a molecular formula of $\mathrm{C}_{15} \mathrm{H}_{10} \mathrm{O}_{5}$. It is a solid compound with a yellow crystalline appearance. ${ }^{67}$ The compound has some biological activities such as antioxidant, anti-inflammatory, antitumor, neuroprotective, cardioprotective and antimicrobial effects. Apigenin has been tested in vivo for different indications including diabetes, amnesia, alzheimer's, depression and insomnia. ${ }^{68}$ In another study, apigenin has been shown to be useful in ameliorating asthma, hypertension, protect liver injury, improved pancreatitis, osteoporosis and arthritis. ${ }^{69}$

Co-administration of apigenin $(25 \mathrm{mg} / \mathrm{kg}$, p.o.) with doxorubicin (12 $\mathrm{mg} / \mathrm{kg}$, i.p.) for 12 days can enhance the cardiac functional parameters 
where there were significant decrease in the levels of cardiac markers induced by doxorubicin. Additionally, there was a significant decrease in cardiac fibrosis significantly as well as apoptotic proteins as compared to doxorubicin only group..$^{70}$ Another group of researchers investigated the cardioprotective effect of apigenin against doxorubicin in male Wistar rats. Animals which received apigenin $(2 \mathrm{mg} / \mathrm{kg})$ administered for 12 days showed significant increase in ejection fraction as well as decreased in $\mathrm{LDH}, \mathrm{CK}-\mathrm{MB}$ levels and left ventricular end diastolic (LVED) thus confirming the reduction in the cardiac damage caused by doxorubicin. ${ }^{71}$

In another study, apigenin was found to inhibit cardiomyocyte apoptosis induced by doxorubicin as confirmed by terminal deoxynucleotidyl transferase dUTP nick end labeling (TUNEL) assay. Following administration of apigenin (125 and $250 \mathrm{mg} / \mathrm{kg}$, i.p., 17 days), the number of apoptotic cells was reduced while the levels of pro-apoptotic proteins Bax and Bcl-2 were also significantly reduced. Additionally, there was significant reduction in the number of autophagic vacuoles using electron microscopy following apigenin administration indicating that it protects against cardiac toxicity as induced by doxorubicin via several different mechanisms. ${ }^{72}$ Furthermore, there were significant improvement in heart contractile function, infarct size, apoptotic rate and reduction in LDH levels. Moreover, it also showed improved cell viability and decreased production of ROS besides acting via ABT-737 (a small mimetic $\mathrm{BH} 3$ small molecule inhibitor) pathway. ${ }^{73}$

\section{Proanthocyanidin}

Proanthocyanidins is a type of phytochemical flavonoid most abundant in the diet such as in grains, spices, fruits, vegetables, nuts and beverages. ${ }^{74,75}$ It is also known as tannins and contribute to the astringent flavor in some foods. Proanthocyanidins are the polymer of flavanols and have flavanol-flavanol linkage which consist of C-C bonds. Proanthocyanidins have antioxidant, anti-inflammatory, anticancer and antidiabetic effects. It also exhibits neuroprotective, antimicrobial, anti-obesity and lipid lowering activities. ${ }^{76,77}$

Proanthocyanidins is a powerful antioxidant since it can cause a reduction in oxidative stress and an increase in the antioxidant enzymes contributing to cardiac damage. Besides, the high number of hydrogen ions $\left(\mathrm{H}^{+}\right)$can block free radical chain reaction causing improved in antioxidant enzymes activity in the cells. Grape seed proanthocyanidins extract (GSPE) helps to ameliorate the cardiac biomarker enzyme levels including AST, ALT, LDH and CK, attributed largely to its antioxidant activity. ${ }^{78}$ Donation of hydrogen atoms to peroxyl radical results in stabilized form of polyphenol phenoxy-radical thus causing a reduction in lipid peroxidation and cardiac enzymes. Finally, the extract $(200 \mathrm{mg} /$ $\mathrm{kg}$, p.o.) can help reduce hydrogen peroxide $\left(\mathrm{H}_{2} \mathrm{O}_{2}\right)$-induced oxidant stress in cardiomyocytes. ${ }^{78}$

Proanthocyanidins is cardioprotective as confirmed histologically in the heart and liver of rats. Following supplementation with grape seed proanthocyanidin extract $(200 \mathrm{mg} / \mathrm{kg}$, p.o., 14 days) together with carboplatin (196 mg/kg, i.p.) which caused cardiac impairment such as 1) cardiac muscle fibers necrosis, 2) degeneration of cardiac tissues and 3) tissue damage, there were more organized cardiac muscle fibers seen.$^{78}$ In another study to determine the cardioprotective effect of proanthocyanidin (100 mg/kg, i.p., 16 days) against doxorubicin $(2 \mathrm{mg} /$ $\mathrm{kg}$, i.p.) in rats, there were fractional shortening and enhanced ejection fraction of the rats' heart seen. Additionally, there were reduced cytoplasmic vacuolization, thus allowing maintainence of cardiac normal structure and morphology indicating that proanthocyanidin extract is cardioprotective. ${ }^{79}$

In another study, male Sprague Dawley rats were used to evaluate the cardioprotective ability of proanthocyanidin in grape seed against doxorubicin-induced cardiotoxicity. Lower cardiac injury was recorded in rats which received a combination of doxorubicin $(10 \mathrm{mg} / \mathrm{kg}$, i.p.) and grape seed (100 mg/kg, p.o., 35 days) based on ameliorated troponin and N-terminal pro-B-type natriuretic peptide (NT-proBNP) levels seen. Moreover, serum malondialdehyde levels were lower in rats which received doxorubicin treatment only indicating that the cardiac protection conferred by proanthocyanidins occurred via reduction in oxidative stress and elevation of cardiac enzyme biomarker. ${ }^{80}$

\section{Indole-3-Carbinol}

Indole-3-carbinol is a chemical compound found in cruciferous vegetables such as cauliflower, broccoli and cabbage. For example, Arabidopsis (rockress) which is a small flowering plant related to mustard or cabbage have long been used as medicine since the time of Pythagoras and Hippocrates. ${ }^{81}$ The compound has $\mathrm{C} 8-\mathrm{H}$ and/or N1-H bonds and $\mathrm{OH}$ groups indole rings which exhibit some antioxidant properties. ${ }^{82}$ Apart from its antioxidant activities, it has anticancer properties by interrupting cancer cell cycles, promoting cell apoptosis, controlling cell division and angiogenesis deregulation of cancer cells. Besides, it is useful in preventing chronic diseases including cardiovascular disease, obesity and diabetes. ${ }^{83}$ To date, the combined treatment of doxorubicin ( $4 \mathrm{mg} / \mathrm{kg}$, i.p.) and indole-3-carbinol (2000 $\mathrm{mg} / \mathrm{L}$ p.o.) has been shown to confer some cardioprotective effects. Beside of lower down tumor volume, tumor malondialdehyde, sphingosine kinase-1 activity, this compound helps to increase the catalase and superoxide dismutase in mice. ${ }^{84}$

Swiss albino mice were used when evaluating the protective effects of indole-3-carbinol ( $20 \mathrm{mg} / \mathrm{kg}$, orally for 15 days) against cardiac toxicity caused by doxorubicin where the compound was found to alleviate oxidative stress by reducing ROS levels and lipid peroxidation as well as enhancing the levels of glutathione and phase-II antioxidant enzymes. ${ }^{85}$ The toxic effect of doxorubicin caused massive structural changes and functional inability of the heart as well as the bone marrow niche. Nevertheless, indole-3-carbinole helped to reduce chromosomal aberrations, micronuclei DNA damage and apoptosis exerted by doxorubicin. ${ }^{86}$

The combination of doxorubicin and indole-3-carbinol caused a reduction in $\mathrm{LDH}, \mathrm{CK}-\mathrm{MB}$ and troponin-I enzymes activities where improved swollen cardiac muscle fibers, interstitial edema and inflammatory infiltration in cardiac tissues were seen. Administration of indole-3-carbinol in a dose-dependent manner also resulted in the reduced production of inflammatory mediators, nitric oxide, TNF- $\alpha$ and IL-10. TNF- $\alpha$ is pro-inflammatory cytokines thought to worsen cardiac damage, remodeling and damage in ventricles. ${ }^{84-86}$

\section{SUMMARY AND FUTURE PERSPECTIVES}

There is an urgent need to uncover the potential of natural products to ameliorate the negative effects of chemotherapeutic drugs. ${ }^{87}$ Overall, most natural products have more than a single "target" and may affect multiple signaling pathways. Therefore, the development of natural products which can target at multiple sites may be useful in ameliorating the cardiotoxicity induced by chemotherapeutic agents. Based on literature reviews, ten natural products (curcumin, mangiferin, naringenin, quercetin, 6-gingerol, lycopene, resveratrol, apigenin, proanthocyanidins and indole-3-carbinol), have demonstrated significant cardioprotective effects on chemotherapyinduced cardiotoxicity as confirmed in both in-vitro and in-vivo studies. Nevertheless, the majority of such findings were confirmed in preclinical studies, and have not really been translated for clinical use. The major impediment to the development of natural productbased cardioprotective adjuvants is attributed to its poor bioavailability in human. ${ }^{88-89}$ Therefore, various synthetic derivatives based on the molecular structures of the active constituents of the natural products are required. Overall, the review indicated that natural products have 
the potential to confer cardioprotective effects against chemotherapyinduced cardiotoxicity.

\section{ACKNOWLEDGEMENT}

The authors would like to thank the Ministry of Higher Education (MOHE) Malaysia for the financial support provided via the Fundamental Research Grant Scheme [FRGS/1/2018/SKK10/ $\mathrm{UNIKL} / 02 / 1]$.

\section{CONFLICTING INTEREST}

The authors declared that they have no competing interests.

\section{REFERENCES}

1. Nygren P. What is cancer chemotherapy? Acta Oncologica. 2001;40:166-74.

2. Fraiser LH, Kanekal S, Kehrer JP. Cyclophosphamide toxicity. Drugs. 1991;42:781-95.

3. Shanholtz C. Acute life-threatening toxicity of cancer treatment. Critical Care Clinics. 2001;17:483-502.

4. Prahalathan C, Selvakumar E, Varalakshmi P. Remedial effect of $D L-\alpha$-lipoic acid against adriamycin induced testicular lipid peroxidation. Molecular and Cellular Biochemistry. 2004;267:209-14.

5. Curigliano G, Cardinale D, Dent S, Criscitiello C, Aseyev O, Lenihan D, et al. Cardiotoxicity of anticancer treatments: epidemiology, detection, and management. CA: A Cancer Journal for Clinicians. 2016;66:309-25.

6. Pai VB, Nahata MC. Cardiotoxicity of chemotherapeutic agents. Drug Safety. 2000;22:263-302

7. Albini A, Pennesi G, Donatelli F, Cammarota R, De Flora S, Noonan DM. Cardiotoxicity of anticancer drugs: the need for cardio-oncology and cardiooncological prevention. Journal of the National Cancer Institute. 2010;102:1425.

8. Shishodia S, Sethi G, Aggarwal BB. Curcumin: Getting back to the roots. Annals of the New York Academy of Sciences. 2005;1056:206-17.

9. Aggarwal BB, Sundaram C, Malani N, Ichikawa H. Curcumin: The Indian solid gold. Advances in Experimental Medicine and Biology. 2007;595:1-75.

10. Katamura M, Iwai-Kanai E, Nakaoka M, Okawa Y, Ariyoshi M, Mita Y, et al Curcumin attenuates doxorubicin-induced cardiotoxicity by inducing autophagy via the regulation of JNK phosphorylation. Journal of Clinical and Experimental Cardiology. 2014;5(9):1000337.

11. Swamy AV, Gulliaya S, Thippeswamy A, Koti BC, Manjula DV. Cardioprotective effect of curcumin against doxorubicin-induced myocardial toxicity in albino rats. Indian Journal of Pharmacology. 2012;44:73

12. Hosseini A, Sahebkar A. Reversal of doxorubicin-induced cardiotoxicity by using phytotherapy: A review. Journal of Pharmacopuncture. 2017;20:243.

13. Sadzuka $Y$, Nagamine M, Toyooka T, Ibuki $Y$, Sonobe T. Beneficial effects of curcumin on antitumor activity and adverse reactions of doxorubicin. International Journal of Pharmaceutics. 2012;432:42-9.

14. Avci H, Epikmen ET, Ipek E, Tunca R, Birincioglu S, Aksit H, et al. Protective effects of silymarin and curcumin on cyclophosphamide-induced cardiotoxicity. Experimental and Toxicologic Pathology. 2017;69:317-27.

15. Chakraborty M, Bhattacharjee A, Kamath JV. Cardioprotective effect of curcumin and piperine combination against cyclophosphamide-induced cardiotoxicity. Indian Journal of Pharmacology. 2017;49:65

16. Bahadır A, Ceyhan A, Gergin OO, Yalçın B, Ulger M, OzyazganTM, et al. Protective effects of curcumin and beta-carotene on cisplatin-induced cardiotoxicity: An experimental rat model. Anatolian Journal of Cardiology. 2018;19:213.

17. Mahendran, S. Molecules of interest - Mangiferin - A review. Annual Research \& Review in Biology 2014;5:307-20

18. Nunez Selles AJ, Daglia M, Rastrelli L. The potential role of mangiferin in cancer treatment through its immunomodulatory, anti-angiogenic, apoptopic, and gene regulatory effects. Biofactors. 2016;42:475-91.

19. Imran M, Arshad MS, Butt MS, Kwon JH, Arshad MU, Sultan MT. Mangiferin: A natural miracle bioactive compound against lifestyle related disorders. Lipids in Health and Disease. 2017;16:84

20. Kiran TGS, Kiran KR, Vinodkumar M. Current pharmacological status of cardioprotective plants against doxorubicin-induced cardiotoxicity: A scoping review. Journal of Pharmaceutical Biology. 2018;3:220-9.

21. Agustini FD, Arozal W, Louisa M, Siswanto S, Soetikno V, Nafrialdi N, et al. Cardioprotection mechanism of mangiferin on doxorubicin-induced rats: Focus on intracellular calcium regulation. Pharmaceutical Biology. 2016;54:1289-97.

22. Bhatt L, Sebastian B, Joshi V. Mangiferin protects rat myocardial tissue against cyclophosphamide induced cardiotoxicity. Journal of Ayurveda and integrative medicine. 2017;8:62-7.
23. Bhatt $L$, Joshi V. Mangifera indica $L$. leaf extract alleviates doxorubicin induced cardiac stress. Journal of Intercultural Ethnopharmacology. 2017;6:284.

24. Prabhu S, Jainu M, Sabitha K, Devi C. Cardioprotective effect of mangiferin on isoproterenol induced myocardial infarction in rats. Indian Journal of Experimental Biology. 2006;44:209-15.

25. Manchope MF, Casagrande R, Verri Jr WA. Naringenin: an analgesic and antiinflammatory citrus flavanone. Oncotarget. 2017;8:3766.

26. Patel K, Singh GK, Patel DK. A review on pharmacological and analytical aspects of naringenin. Chinese Journal of Integrative Medicine. 2018;24:551-60.

27. Zeng $W$, Jin L, Zhang F, Zhang $C$, Liang $W$. Naringenin as a potential immunomodulator in therapeutics. Pharmacological Research. 2018;135:122-6.

28. Salehi B, Fokou PVT, Sharifi-Rad M, Zucca P, Pezzani R, Martins N, et al. The therapeutic potential of naringenin: A review of clinical trials. Pharmaceuticals. 2019;12:11.

29. Subburaman S, Ganesan K, Ramachandran M. Protective role of naringenin against doxorubicin-induced cardiotoxicity in a rat model: histopathology and mRNA expression profile studies. Journal of Environmental Pathology, Toxicology and Oncology. 2014;33:363-76.

30. Arafa HM, Abd-Ellah MF, Hafez HF. Abatement by naringenin of doxorubicininduced cardiac toxicity in rats. Journal of the Egyptian National Cancer Institute. 2005;17:291A300

31. Han X, Pan J, Ren D, Cheng Y, Fan P, Lou H. Naringenin-7-O-glucoside protects against doxorubicin-induced toxicity in $\mathrm{H} 9 \mathrm{c} 2$ cardiomyocytes by induction of endogenous antioxidant enzymes. Food and Chemical Toxicology. 2008;46:3140-6.

32. Kwatra M, Kumar V, Jangra A, Mishra M, Ahmed S, Ghosh P, et al. Ameliorative effect of naringin against doxorubicin-induced acute cardiac toxicity in rats. Pharmaceutical Biology. 2016;54:637-47.

33. Jagetia GC, Reddy TK. The grape fruit flavonone naringin protects mice against doxorubicin-induced cardiotoxicity. Journal of Molecular Biochemistry. 2014;3:34-49.

34. David AVA, Arulmoli R, Parasuraman S. Overviews of biological importance of quercetin: A bioactive flavonoid. Pharmacognosy Reviews. 2016;10:84.

35. LiY, Yao J, Han C, Yang J, Chaudhry MT, Wang S, et al. Quercetin, inflammation and immunity. Nutrients. 2016;8:167.

36. Domanska U, Wisniewska A, Dąbrowski Z, Więckowski M. Ionic liquids as efficient extractants for quercetin from red onion (Allium cepa L.). Journal of Applied Solution Chemistry and Modeling. 2018;7:21-38.

37. Sharma A, Kashyap D, Sak K, Tuli HS, Sharma AK. Therapeutic charm of quercetin and its derivatives: a review of research and patents. Pharmaceutical Patent Analyst. 2018;7:15-32.

38. Zakaria N, Khalil SR, Awad A, Khairy GM. Quercetin reverses altered energy metabolism in the heart of rats receiving adriamycin chemotherapy. Cardiovascular Toxicology. 2018;18:109-19.

39. Abdullah MA, Abd AA, Baker SA. A biochemical study of the effect of quercetin on cisplatin induced rat tissues toxicity. American Journal of Biochemistry. 2018;8:87-92.

40. Chen $X$, Peng $X$, Luo $Y$, You J, Yin D, Xu Q, et al. Quercetin protects cardiomyocytes against doxorubicin-induced toxicity by suppressing oxidative stress and improving mitochondrial function via 14-3-3 $\gamma$. Toxicology Mechanisms and Methods. 2019;29:344-54.

41. Qureshi WA, Zhao $R$, Wang $H$, Ji T, Ding $Y$, Ihsan A, et al. Co-delivery of doxorubicin and quercetin via $\mathrm{MPEG}-\mathrm{PLGA}$ copolymer assembly for synergistic anti-tumor efficacy and reducing cardio-toxicity. Science Bulletin. 2016;61:168998.

42. Al Fatease A, Shah V, Nguyen DX, Cote B, LeBlanc N, Rao DA, et al Chemosensitization and mitigation of Adriamycin-induced cardiotoxicity using combinational polymeric micelles for co-delivery of quercetin/resveratro and resveratrol/curcumin in ovarian cancer. Nanomedicine: Nanotechnology, Biology and Medicine. 2019;19:39-48.

43. Da Silva TA, Gomes JHR, de Bulhões LCG, Neto R, Junior IDB, de Moura M, et al. Therapeutic potential of quercetin based on nanotechnology: A review. Revista Virtual de Química. 2019;11:1405-16.

44. Jiang $Y$, Huang M, Wisniewski M, Li H, Zhang M, Tao $X$, et al. Transcriptome analysis provides insights into gingerol biosynthesis in ginger (Zingiber officinale). The plant genome. 2018;11:1-11.

45. Yusof YAM. Gingerol and its role in chronic diseases. Drug Discovery from Mother Nature: Springer, pp;2016

46. Wang $Q$, Wei $Q$, Yang $Q$, Cao X, Li Q, Shi F, et al. A novel formulation of [6]-gingerol: Proliposomes with enhanced oral bioavailability and antitumor effect. International Journal of Pharmaceutics. 2018;535:308-15.

47. Ananthanarayanan Ajith T, Hema U, Aswathi S. Zingiber officinale Roscoe ameliorates anticancer antibiotic doxorubicin-induced acute cardiotoxicity in rat. Journal of Experimental Therapeutics \& Oncology. 2016;11:171-5. 
48. El-Hawwary AA, Omar NM. The influence of ginger administration on cisplatininduced cardiotoxicity in rat: Light and electron microscopic study. Acta Histochemica. 2019;121:553-62.

49. Shalaby NMM, Mahmoud ARH, Ali NE-SM, Ibrahem NE, Mekawy NHAH. The possible protective effect of Zingiber officinale extract on cyclophosphamideinduced cardiotoxicity in adult male albino rats. Journal of Toxicology and Environmental Health Sciences. 2019;11:38-49.

50. Al-Abbasi FA, Alghamdi EA, Baghdadi MA, Alamoudi AJ, El-Halawany AM, ElBassossy HM, et al. Gingerol synergizes the cytotoxic effects of doxorubicin against liver cancer cells and protects from its vascular toxicity. Molecules. 2016;21:886

51. Ojha S, AlTaee H, Goyal S, Mahajan UB, Patil CR, Arya D, et al. Cardioprotective potentials of plant-derived small molecules against doxorubicin associated cardiotoxicity. Oxidative Medicine and Cellular Longevity. 2016;2016:1-19.

52. Yang $X$, Liu N, Li $X$, Yang $Y$, Wang $X$, Li L, et al. A review on the effect of traditional chinese medicine against anthracycline-induced cardiac toxicity. Frontiers in Pharmacology. 2018;9:444

53. Mozos I, Stoian D, Caraba A, Malainer C, Horbańczuk J, Atanasov A. Lycopene and vascular health. Frontiers in Pharmacology. 2018;9:521.

54. Sen S. The chemistry and biology of lycopene: Antioxidant for human health. International Journal of Advancement in Life Sciences Research. 2019:8-14.

55. Muller L, Caris-Veyrat C, Lowe G, Bohm V. Lycopene and its antioxidant role in the prevention of cardiovascular diseases - A critical review. Critical Reviews in Food Science and Nutrition. 2016;56:1868-79.

56. Zhu J, Hu Q, Shen S. Enhanced antitumor efficacy and attenuated cardiotoxicity of doxorubicin in combination with lycopene liposomes. Journal of Liposome Research. 2020;30:37-44

57. Shalaby NMM, Soliman WI. Comparative study on the effects of lycopene and saffron on doxorubicin-induced cardiotoxicity in adult male albino rats: A histological and biochemical assessment. The Egyptian Journal of Forensic Sciences and Applied Toxicology. 2017;17(1):237-59.

58. 58. Colica C, Milanovic M, Milic N, Aiello V, De Lorenzo A, Abenavoli L. A systematic review on natural antioxidant properties of resveratrol. Natural Product Communications. 2018;13(9):1195-203

59. Berman AY, Motechin RA, Wiesenfeld MY, Holz MK. The therapeutic potential of resveratrol: A review of clinical trials. NPJ Precision Oncology. 2017;1:1-9.

60. Gu J, Hu W, Song ZP, Chen YG, Zhang DD, Wang CQ. Resveratrol-induced autophagy promotes survival and attenuates doxorubicin-induced cardiotoxicity. International Immunopharmacology. 2016;32:1-7.

61. Abdelgawad IY, Grant MK, Zordoky BN. Leveraging the cardio-protective and anticancer properties of resveratrol in cardio-oncology. Nutrients. 2019;11:627.

62. Liu MH, Lin XL, Guo DM, Zhang Y, Yuan C, Tan TP, et al. Resveratrol protects cardiomyocytes from doxorubicin-induced apoptosis through the AMPK/P53 pathway. Molecular Medicine Reports. 2016;13:1281-6.

63. Alanazi AM, Fadda L, Alhusaini A, Ahmad R, Hasan IH, Mahmoud AM. Liposomal resveratrol and/or carvedilol attenuate doxorubicin-induced cardiotoxicity by modulating inflammation, oxidative stress and S100A1 in rats. Antioxidants. 2020;9(2):159

64. Zhang L, Zhu K, Zeng H, Zhang J, Pu Y, Wang Z, et al. Resveratrol solid lipid nanoparticles to trigger credible inhibition of doxorubicin cardiotoxicity. International Journal of Nanomedicine. 2019;14:6061-71.

65. Matsumura N, Zordoky BN, Robertson IM, Hamza SM, Parajuli N, Soltys C-LM, et al. Co-administration of resveratrol with doxorubicin in young mice attenuates detrimental late-occurring cardiovascular changes. Cardiovascular Research. 2018;114:1350-9.

66. Shukla S, Gupta S. Apigenin: A promising molecule for cancer prevention. Pharmaceutical Research. 2010;27:962-78.

67. Ali F, Rahul, Naz F, Jyoti S, Siddique YH. Health functionality of apigenin: A review. International Journal of Food Properties. 2017;20:1197-238.

68. Salehi B, Venditti A, Sharifi-Rad M, Kregiel D, Sharifi-Rad J, Durazzo A, et al. The therapeutic potential of apigenin. International Journal of Molecular Sciences. 2019;20:1305
69. Zhou X, Wang F, Zhou R, Song X, Xie M. Apigenin: A current review on its beneficial biological activities. Journal of Food Biochemistry. 2017;41:e12376.

70. Zare MFR, Rakhshan K, Aboutaleb N, Nikbakht F, Naderi N, Bakhshesh M, et al. Apigenin attenuates doxorubicin induced cardiotoxicity via reducing oxidative stress and apoptosis in male rats. Life Sciences. 2019;232:116623.

71. Rakhshan K, Aboutaleb N, Nikbakht F, Bakhshesh M, Azizi Y. Apigenin improves myocardial function and attenuates cardiotoxicity induced by doxorubicin in male rats. Yafteh. 2018;20:85-98.

72. YuW, Sun H, Zha W, Cui W, Xu L, Min Q, et al. Apigenin attenuates adriamycininduced cardiomyocyte apoptosis via the PI3K/AKT/mTOR pathway. EvidenceBased Complementary and Alternative Medicine. 2017;2017:1-9.

73. Chen $\mathrm{C}$, He H, Luo Y, Zhou M, Yin D, He M. Involvement of Bcl-2 signal pathway in the protective effects of apigenin on anoxia/reoxygenation-induced myocardium injury. Journal of Cardiovascular Pharmacology. 2016;67:152-63.

74. Blade C, Aragonès G, Arola-Arnal A, Muguerza B, Bravo Fl, Salvadó MJ, et al. Proanthocyanidins in health and disease. Biofactors. 2016;42:5-12.

75. Haytowitz D, Wu X, Bhagwat S. USDA Database for the proanthocyanidin content of selected foods Release 2.1. US Department of Agriculture, Agricultural Service. Nutrient Data Laboratory Home Page: Retrieved from http://www. ars. usda. gov/nutrientdata/flav (accessed on 19 May 2019). 2018:1-46.

76. Zhu F. Proanthocyanidins in cereals and pseudocereals. Critical Reviews in Food Science and Nutrition. 2019;59:1521-33.

77. Rauf A, Imran M, Abu-Izneid T, Patel S, Pan X, Naz S, et al. Proanthocyanidins: A comprehensive review. Biomedicine \& Pharmacotherapy. 2019;116:108999.

78. Yousef MI, Mahdy MA, Abdou HM. The potential protective role of grape seed proanthocyanidin extract against the mixture of carboplatin and thalidomideinduced hepatotoxicity and cardiotoxicity in male rats. Preventive Medicine. 2019;2:1-7.

79. Razmaraii N, Babaei H, Nayebi AM, Assadnassab G, Helan JA, Azarmi Y. Cardioprotective effect of grape seed extract on chronic doxorubicin-induced cardiac toxicity in Wistar rats. Advanced Pharmaceutical Bulletin. 2016;6:423.

80. Adıyaman MS, Adıyaman OA, Dagl AF, Karahan MZ, Kaya I, Dagl MN. Effects of grapeseed extract on doxorubicin-induced cardiotoxicity in rats. Herz. 2020:1-6.

81. Katz E, Nisani S, Chamovitz DA. Indole-3-carbinol: A plant hormone combatting cancer. F1000Research. 2018;7:689.

82. Vo QV, Mechler A. In silico study of the radical scavenging activities of natural indole-3-carbinols. Journal of Chemical Information and Modeling. 2019;60:31621.

83. Licznerska B, Baer-Dubowska W. Indole-3-carbinol and its role in chronic diseases. Advances in Experimental Medicine and Biology. 2016;928:131-54.

84. Adwas AA, Elkhoely AA, Kabel AM, Abdel-Rahman MN, Eissa AA. Anti-cancer and cardioprotective effects of indol-3-carbinol in doxorubicin-treated mice. Journal of Infection and Chemotherapy. 2016;22:36-43.

85. Hajra S, Patra AR, Basu A, Bhattacharya S. Prevention of doxorubicin (DOX)induced genotoxicity and cardiotoxicity: Effect of plant derived small molecule indole-3-carbinol $(\mathrm{I} 3 \mathrm{C})$ on oxidative stress and inflammation. Biomedicine \& Pharmacotherapy. 2018;101:228-43.

86. Hajra S, Patra AR, Basu A, Saha P, Bhattacharya S. Indole-3-Carbinol (I3C) enhances the sensitivity of murine breast adenocarcinoma cells to doxorubicin (DOX) through inhibition of NF- $\kappa \beta$, blocking angiogenesis and regulation of mitochondrial apoptotic pathway. Chemico-biological Interactions. 2018;290:1936.

87. Selim MS, Amer SK, Mohamed SS, Mounier MM, Rifaat HM. Production and characterisation of exopolysaccharide from Streptomyces carpaticus isolated from marine sediments in Egypt and its effect on breast and colon cell lines. Journal of Genetic Engineering and Biotechnology. 2018;16:23-8.

88. Anand P, Kunnumakkara AB, Newman RA, Aggarwal BB. Bioavailability of curcumin: Problems and promises. Molecular Pharmaceutics. 2007;4:807-18.

89. Novelle MG, Wahl D, Dieguez C, Bernier M, de Cabo R. Resveratrol supplementation: where are we now and where should we go? Ageing Research Reviews. 2015;21:1-15. 


\section{GRAPHICAL ABSTRACT}

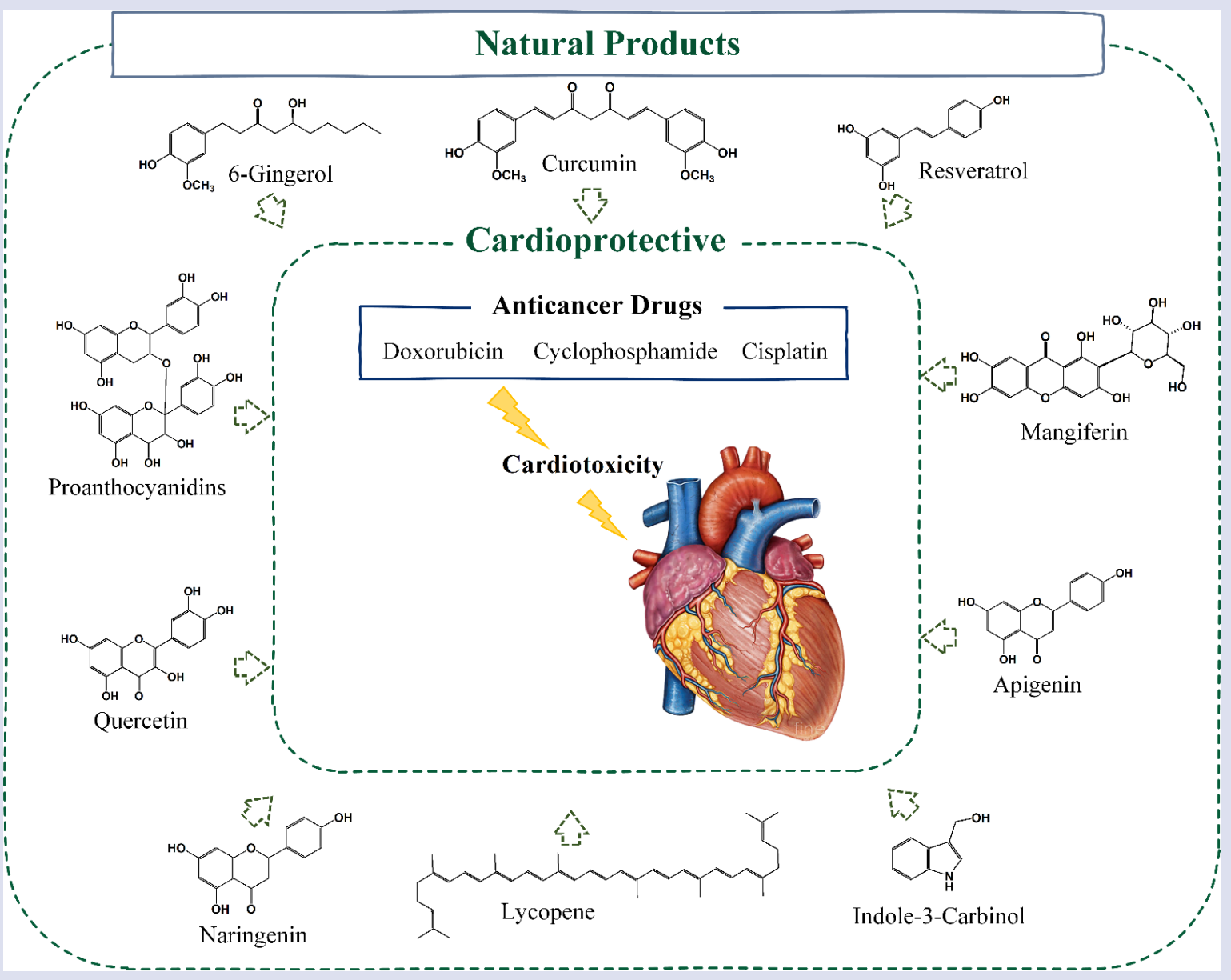

\section{ABOUT AUTHORS}

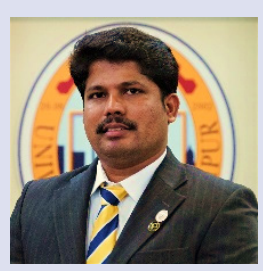

Mahendran Sekar is currently working as Associate Professor in Department of Pharmaceutical Chemistry, Faculty of Pharmacy and Health Sciences, Universiti Kuala Lumpur Royal College of Medicine Perak, Ipoh, Perak, Malaysia. His research is mainly in the field of drug discovery and development of natural products. His research interest is isolation of active constituents from medicinal plants and study about its biological properties.

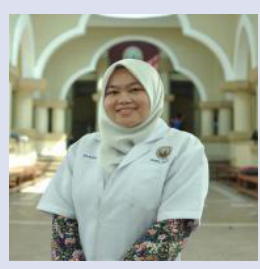

Siti Nurul Najiha Othman is currently pursuing Master of Pharmacy (By Research) at Universiti Kuala Lumpur Royal College of Medicine Perak, Ipoh, Perak, Malaysia. Her research interest is isolation of active constituents from medicinal plants and study about its biological properties.

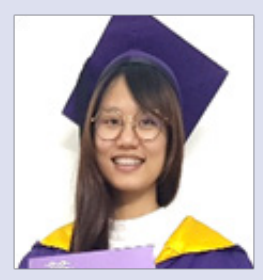

PeiTeng Lum is currently pursuing Ph.D in Pharmacy at Universiti Kuala Lumpur Royal College of Medicine Perak, Ipoh, Perak, Malaysia. Her research interest is isolation of active constituents from medicinal plants and study about its effect on neurodegenerative disorders. 
Siew Hua Gan is currently working as a Professor \& Head of School of Pharmacy, Monash University Malaysia, Selangor, Malaysia. Gan is a trainer for scientific writing and professional development programmes including presentation skills, viva defense \& time management. She has been conducting workshops in several institutions in Malaysia (since 2005) as well as in India (in 2017). Her area of specialisations include pharmacogenetics, toxicology and natural products.

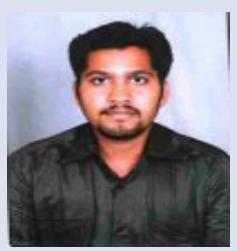

Shankar Mani is currently working as Associate Professor in Sri Adichunchanagiri College of Pharmacy, Adichunchanagiri University, Karnataka, India. His research interest is mainly in synthesis of medicinally important compounds and study about its biological properties. He also interest in the research of drug discovery and development of natural products.

Cite this article: Othman SNN, Lum PT, Gan SH, Mani S, Sekar M. Protective Effect of Natural Products against ChemotherapyInduced Cardiotoxicity: A Review. Pharmacogn J. 2020;12(5):1180-9. 